\title{
EFICIENCIA DE LA INCLUSIÓN FINANCIERA EN COLOMBIA, 2014*
}

\author{
Recibido: 20 de diciembre de 2016 • Aprobado: 14 de julio de 2017 \\ DOI: $10.22395 /$ seec.v20n44a4
}

\section{Gloria Isabel Rodríguez Lozano**}

\section{RESUMEN}

Existe una correlación bastante alta entre la exclusión de los servicios ofrecidos por el sector financiero formal y la pobreza. Es por esto que en este trabajo se mide, para el año 2014, la eficiencia de la inclusión financiera en Colombia. En el análisis se incluye a los bancos, las compañías de financiamiento y las cooperativas financieras, los cuales son estudiados de manera no global, mediante la aplicación del análisis envolvente de datos, metodología no paramétrica de programación lineal que mediante un doble proceso de optimización genera indicadores de eficiencia. En este trabajo se da respuesta a los siguientes interrogantes: ¿Qué tipo de entidades son eficientes y en qué número? ¿Cómo es la distribución de las entidades según el rango de índice de eficiencia? ¿Hay algún grupo que se diferencie? Los resultados revelan que solo el $25 \%$ de las instituciones analizadas son eficientes.

\section{PALABRAS CLAVE}

Inclusión financiera; eficiencia de la inclusión financiera; instituciones financieras; bancos y otras instituciones de crédito; análisis envolvente de datos; Colombia.

\section{CLASIFICACIÓN JEL} G20, G21

\section{CONTENIDO}

Introducción; 1. Inclusión financiera. Definición; 2. Medición de la inclusión financiera; 3. Metodología; 4. Revisión de la literatura actual; 5. Diseño metodológico; 6. Resultados; 7. Conclusiones; Bibliografía; Anexo.

\footnotetext{
* Este artículo de investigación es producto del proyecto titulado Estudio de la eficiencia y la productividad de los sectores de la economía colombiana, adelantado en la Universidad Nacional de Colombia, con financiación de la Facultad de Ciencias Económicas, Universidad Nacional de Colombia, Bogotá. Fue desarrollado entre enero de 2015 y noviembre de 2016.

" Administradora de Empresas, Doctora en Ciencias Económicas, Universidad Nacional de Colombia Bogotá, Colombia. Magíster en Ingeniería Industrial, Universidad de los Andes, Bogotá, Colombia. Profesora asociada, Facultad de Ciencias Económicas, Universidad Nacional de Colombia, Bogotá, Colombia. Ciudad Universitaria, Edificio 311, Oficina 308, Bogotá, Colombia. Correo electrónico: girodriguezl@unal.edu.co
} 


\section{EFFICIENCY OF FINANCIAL INCLUSION IN COLOMBIA, 2014 ABSTRACT}

There is a very strong correlation between exclusion of services offered by formal financial sector and poverty. For this reason, this work measures, for year 2014, the efficiency of the financial inclusion in Colombia. The analysis includes banks, financing companies, and financial credit unions which are not studied in a global manner by applying the data envelopment analysis, a non-parametric linear programming methodology which, through a double optimization process, generates efficiency indicators. This research gives an answer to the following questions: What types of entities are efficient and how many of them? How is the distribution of entities according to the efficiency index range? Is there any distinguishing group? Results reveal that only $25 \%$ of the institutions subject to analysis are efficient.

\section{KEY WORDS}

Financial inclusion; financial inclusion efficiency; financial institutions; banks and other credit institutions; data envelopment analysis; Colombia.

\section{JEL CLASSIFICAITON}

G20, G21

\section{CONTENT}

Introduction; 1. Financial inclusion. Definition; 2. Measurement of financial inclusion; 3. Methodology; 4. Revision of current literature; 5. Methodological method; 6. Results; 7. Conclusions; Bibliography; Annex.

\section{EFICIÊNCIA DA INCLUSÃO FINANCEIRA NA COLÔMBIA, 2014 RESUMO}

Existe uma correlação muito alta entre a exclusão dos serviços oferecidos pelo setor financeiro formal e a pobreza. Por isso, neste trabalho, mede-se, para o ano 2014, a eficiência da inclusão financeira na Colômbia. Na análise, incluem-se os bancos, as companhias de financiamento e as cooperativas financeiras, que são estudados de maneira não global, mediante a aplicação da análise envolvente de dados e pela metodologia não paramétrica de programação linear que, por meio de um duplo processo de otimização, gera indicadores de eficiência. Neste trabalho, dá-se resposta às seguintes questões: que tipo de entidades são eficientes e em que número? Como é a distribuição das entidades segundo o intervalo de índice de eficiência? Há algum grupo que se diferencie? Os resultados demonstram que somente $25 \%$ das instituições analisadas são eficientes.

\section{PALAVRAS-CHAVE}

Inclusão financeira; eficiência da inclusão financeira; instituições financeiras; bancos e outras instituições de crédito; análise envolvente de dados; Colômbia.

\section{CLASSIFICAÇÃO JEL}

G20, G21

\section{CONTEÚDO}

Introdução; 1. Inclusão financeira. Definição; 2. Medição da inclusão financeira; 3. Metodologia; 4. Revisão da literatura atual; 5. Desenho metodológico; 6. Resultados: 7. Conclusões; Bibliografia; Anexo. 


\section{INTRODUCCIÓN}

La inclusión financiera es un tema de gran importancia, con prioridad en los países en desarrollo, idea resaltada por el Banco Central del Ecuador (BCE, 2012, p. 10), el cual se refiere a la inclusión financiera como un fenómeno global en ascenso.

De la misma manera se considera que existe una correlación bastante alta entre la exclusión de los servicios ofrecidos por el sector financiero formal y la pobreza; tanto es así que Burgess y Pande (2005, p. 790), en investigación que realizaron en la India, lograron demostrar que ante la apertura de sucursales en lugares rurales sin servicios bancarios se dio una reducción en la pobreza rural en estas zonas. Ahora bien, Zhan y Sherraden (2011, p. 852) demostraron, en investigación realizada en Estados Unidos, que al existir regulaciones financieras más inclusivas, las familias de bajos ingresos están motivadas a ahorrar para pagar la educación universitaria de sus hijos. De otra manera, Bruhn y Inessa (2016) determinaron que la ampliación del acceso a la financiación formal a las personas de bajos ingresos trae un efecto positivo en el aumento del empleo total, en el ingreso promedio y en la creación y supervivencia de las empresas informales.

Desde otro ángulo, Roa (2014, p. 21) llega a la conclusión de que el contar con un mejor acceso a los mercados financieros formales disminuye los riesgos inherentes a la participación en el sector financiero informal; y para nadie es un secreto que la población más vulnerable a acceder a servicios financieros informales es la población de más bajos recursos; de otra parte Cull, Demirgüç-Kunt, y Lyman (2012, p. 4) aseguran que "Los sectores con gran capacidad financiera no son precisamente los más inclusivos, ya que el acceso financiero se inclina sobre todo hacia los ricos".

Ahora bien, en una investigación de los prestatarios del programa Compartamos en México, realizado por Angelucci, Karlan y Zinman (2013, p. 3) se llegó a la conclusión de que al acceso al crédito formal reporta un panorama general positivo, pues existe "disminución de la depresión, aumento de la confianza en los demás y aumento del poder de decisión de la mujer en el hogar". Como una de las conclusiones de su trabajo de investigación Cull, Ehrbeck y Holle (2014a, p. 1) explicitan que un gran número de hogares pobres saca su subsistencia de la economía informal. Para De Koker (2013, p. 267) una gran parte de la población en los países en desarrollo opera en la economía informal a base de dinero en efectivo, ahorran, y tienen acceso a dinero y a crédito a través de servicios financieros no regulados y no supervisados.

Para Cull, Ehrbeck y Holle (2014b, p. 3) los hogares con bajos recursos casi siempre viven y trabajan en el sector informal de la economía, y sería muy beneficioso para ellos el tener acceso y poder usar los servicios financieros formales. Según 
Friedline y Rauktis (2014, p. 593) la inclusión financiera es un paso para la creación de potentes ayudas para mejorar el bienestar de los jóvenes a lo largo de su vida. De otra manera, la inclusión financiera es un componente clave de la capacidad financiera, un enfoque que se refiere a la combinación de la información acerca del acceso a servicios financieros básicos según Johnson y Sherraden (2007, p. 121).

Entre tanto, Roa (2013, p. 306) considera que tanto las instituciones financieras como los gobiernos deben diseñar programas, tales como programas de educación financiera, con el fin de promover la inclusión financiera y animar a la gente a tomar mejores decisiones financieras, en especial en las economías en desarrollo.

Diferentes autoridades en el ámbito mundial han establecido que la inclusión financiera es de la mayor importancia en cuanto al desarrollo de los países; por ejemplo, el Grupo de los Veinte (G-20) instauró lo correspondiente para que este tema fuese tratado como uno de los principales en la cumbre que se desarrolló en Pittsburgh en el año 2009; como prueba de su importancia, según el Banco Mundial (2016, p. 13 ), para el año 2013 más de cincuenta países hicieron declaraciones públicas en las cuales se responsabilizaban por desplegar estrategias de inclusión financiera a través de sus órganos regulatorios y normativos. También en el año 2009 se crea la Alianza para la Inclusión Financiera (AFI, Alliance for Financial Inclusion), red global de responsables de política financiera en países desarrollados y en desarrollo, que hacen esfuerzos para que los pobres aumenten su acceso a los diferentes servicios financieros, según AFI (2016a, p. 6).

Para AFI (2015, p. 12) existen 54 comités institucionales que, a su vez, involucran el $63 \%$ de los miembros de AFI, y que incluyen 125 instituciones regulatorias de política financiera de 96 países en desarrollo.

En la actualidad casi todos los organismos internacionales a cargo de la inclusión financiera tienen definiciones e indicadores; pero tanto de las unas como de los otros se establece el principio multidimensional del concepto de inclusión financiera; este principio contempla factores que corresponden tanto a la oferta de productos y servicios financieros como a la demanda; pero siempre se utilizan indicadores tradicionales caracterizados por su variedad y su alto número.

En este trabajo se analiza la situación de la inclusión financiera en Colombia para el año 2014, desde un diferente punto de vista: la eficiencia relativa; las entidades financieras son el objeto específico, no global, de análisis. Por esto se considera que esta investigación es pionera. Se generan los indicadores de eficiencia relativa de las cooperativas financieras, de los bancos y de las compañías de financiamiento. La metodología que se utiliza es el Análisis Envolvente de Datos (Data Envlopment 
Analysis-DEA-), metodología de programación lineal que mediante un doble proceso de optimización proporciona un único índice de eficiencia relativa para cada una de las unidades estudiadas, con la fortaleza de analizar las variables en un escenario de utilización de multi-recursos y de obtención de multi-salidas. Se da respuesta a los siguientes interrogantes: ¿Qué tipo de entidades son eficientes y en qué número? ¿Cómo es la distribución de las entidades según el rango de índice de eficiencia? ¿Hay algún grupo que se diferencie?

\section{INCLUSIÓN FINANCIERA. DEFINICIÓN}

En un inicio se utilizó el término bancarización para hacer referencia a la inclusión financiera, término que fue definido por Morales y Yáñez (2006, p. 9) así: "el establecimiento de relaciones estables y amplias entre las instituciones financieras y sus usuarios, respecto a un conjunto de servicios financieros disponibles"; aunque es un concepto que va más allá de las relaciones con los bancos, en términos generales se prestaba para confusiones, pues la gente del común lo asociaba con las relaciones con los bancos.

Hoy en día en el ámbito internacional se maneja el término de inclusión financiera, con múltiples definiciones de múltiples autores y organismos; por ejemplo, para el Grupo Consultivo de Ayuda a los Pobres (CGAP, por sus siglas en inglés) es un entorno en el que los adultos económicamente activos tienen real acceso a los servicios financieros formales, dentro de ellos: seguros, pagos, ahorro y crédito CGAP (2011, p. 9). Considera el mencionado acceso como aquello que tiene que ver con la prestación de un servicio de manera conveniente y, además, responsable, a un costo tal que el cliente pueda asumir y que sea sostenible para el proveedor, y que de esto resulte que las personas excluidas tengan acceso a los servicios financieros formales y, por lo tanto, que no tengan que acceder a opciones informales.

De otra manera, hay definiciones que resaltan por su sencillez, como la de Bayero (2015, p. 49): es la incapacidad de acceder a servicios financieros apropiados. La de Allen et al. (2016, p. 2): es el uso de los servicios financieros formales. Para Appleyard (2011, p. 250), la inclusión financiera es donde los empresarios y las empresas pueden abrir una cuenta bancaria y tener acceso a facilidades de crédito asequibles como un sobregiro o tarjeta de crédito.

La definición siguiente es algo más compleja, del Committee on Financial Inclusion (CFI, 2008, p. 33): proceso de garantizar el poder acceder a los servicios financieros y de crédito, de forma oportuna y adecuada, cuando lo necesiten los sectores más débiles de una economía y los grupos de bajos ingresos a un costo asequible. Para Thorat (2006, p. 240), la inclusión financiera implica la prestación de servicios 
financieros asequibles, servicios como el acceso a los pagos y las instalaciones de remesas, ahorros, préstamos y servicios de seguros por parte de los sistemas financieros formales a quienes tienden a ser excluidos.

Para Dasgupta (2009, p. 41) inclusión financiera es un proceso de hacer que los servicios financieros formales sean accesibles y asequibles para todos; los servicios financieros no significan la provisión de crédito por sí solo, es el suministro de todos los demás servicios, en especial, ahorro, seguros e instalaciones de remesas.

Gwalani y Parkhi (2014, p. 372) consideran que es el proceso de garantizar el acceso a los servicios financieros y de crédito de tal manera que sean oportunos y adecuados cuando lo requieran grupos vulnerables, tales como los sectores más débiles y grupos de bajos ingresos a un precio asequible.

Por su parte, la Organización para la Cooperación y el Desarrollo Económicos (OCDE), por medio del sub-grupo Red Internacional de Educación Financiera (INFE), define la inclusión financiera como aquel proceso en el cual se promociona el acceso oportuno y adecuado a un vasto abanico de servicios y productos financieros que estén regulados y que se extienda su uso a todos los segmentos de la sociedad por medio de la aplicación de estrategias innovadoras hechas a la medida, que incluyan actividades tanto de sensibilización como de educación financiera, y que promuevan el bienestar financiero, la inclusión económica y la social; lo anterior según García et al. (2013, p. 18).

La AFI considera que cuando se habla de inclusión financiera se deben tener en cuenta cuatro aspectos: el uso, el acceso, el bienestar y la calidad. Consideran el acceso como "las capacidades para poder usar los servicios financieros formales disponibles"; el uso como "permanencia y profundidad del uso de servicios y productos financieros". De otra manera consideran que la calidad estudia en qué medida los atributos de los servicios y productos se acomodan a las necesidades de los usuarios y en qué medida el desarrollo de productos ha tomado en cuenta estas necesidades. Y el bienestar tiene en cuenta el impacto positivo que un determinado servicio financiero tiene en la vida de los demandantes (AFI, 2011, p. 15).

Para el Consejo Nacional de Inclusión Financiera de México (CNIF, 2012, p. 8) la inclusión financiera es "el acceso y uso de servicios financieros desde una regulación apropiada que garantice esquemas de protección al consumidor y promueva la educación financiera para mejorar las capacidades financieras de todos los segmentos de la población".

Desde otro ángulo, Kapoor (2014, p. 37) considera que la inclusión financiera es ayudar a las personas a mejorar sus vidas, reducir las desigualdades y con ello superar 
la pobreza. Y para Kear (2013, p. 930), desde el punto de vista de las instituciones financieras, es la extensión del gobierno financiero a nuevas poblaciones.

Para Villacorta y Reyes (2012, p. 5) la inclusión financiera es una situación en la cual "las diferentes personas acceden y usan un grupo de servicios financieros que incluyen servicios de crédito, ahorros, pagos y seguros". Y para Sarma y Pais (2011, p. 613), la inclusión financiera se refiere a un proceso que garantiza la facilidad de acceso, la disponibilidad y el uso del sistema financiero formal para todos los miembros de una economía.

En Brasil, en el informe anual de 2011 de inclusión financiera, el Banco Central de Brasil (BCB, 2011, p. 09) define a esta en términos que tienen que ver con adelantar el acceso y uso eficientes de la población a diferentes servicios financieros que se consideran apropiados a sus diferentes necesidades, y que contribuyen a mejorar su calidad de vida.

Según lo expuesto con anterioridad, se puede establecer que más que una definición como tal, se debe tener en cuenta el concepto multidimensional de inclusión financiera.

\section{MEDICIÓN DE LA INCLUSIÓN FINANCIERA}

El G-20 reconoce el papel clave de la inclusión financiera en la lucha contra la pobreza y en la búsqueda de un desarrollo inclusivo, de tal manera que propone se mida en tres dimensiones: (i) el acceso a los servicios financieros; (ii) el uso de los servicios financieros; y (iii) la calidad de los productos y la prestación de servicios.

Dado que, tanto los datos de la oferta como los de la demanda deben hacer parte de una visión global de la inclusión financiera, GPFI (2016, p. 1) propone un grupo de 24 indicadores (8 de ellos propuestos en el año 2016): adultos con una cuenta, número de cuentas, adultos con crédito en una institución regulada, adultos con seguro, transacciones sin dinero en efectivo, adultos con pagos digitales, pagos usando teléfonos móviles (desde una cuenta), pagos usando Internet, pago a través de una tarjeta bancaria, pago mediante cuenta, alta frecuencia de uso de cuenta, propensión al ahorro, empresas en bancos formales, empresas con préstamo pendiente o línea de crédito en instituciones reguladas, pagos digitales desde o hacia empresas, puntos de servicio, tarjeta débito, puntos de servicio de empresas, inter-operatividad de los puntos de servicio, conocimiento financiero, comportamiento financiero, información de requisitos, resolución de reclamos y barreras crediticias. 
La AFI (2016b, p. 3) en su metodología plantea cuatro dimensiones para adelantar la medición en cada país: acceso, uso, calidad y bienestar. Ahora bien, en estudios realizados por The Economist Intelligence Unit (2014, p. 8), se evalúa el ambiente normativo con base en 12 indicadores. De otra manera, World Bank Group (2015, p. 10) presenta una metodología con 9 indicadores y 41 sub-indicadores para cada país.

Para la Comisión Económica para América Latina-CEPAL-, de acuerdo con la publicación hecha por Vera y Titelman (2013, p. 12), algunos de los indicadores usados para medir la inclusión financiera son aquellos que miden el acceso a los servicios financieros: porcentaje de adultos que dicen tener una cuenta en una institución financiera formal, porcentaje de individuos mayores de 15 años que utilizan tarjetas débito o crédito, porcentaje de empresas que han obtenido una línea de crédito o un préstamo diferenciados por el tamaño.

Dado que el objetivo del Brookings Financial and Digital Inclusion Project-FDIP- es proporcionar a los legisladores, al sector privado, a los representantes de organizaciones no gubernamentales, y al público en general la información que puede ayudar a mejorar la inclusión financiera en sus respectivos países, ha diseñado 33 indicadores que abarcan cuatro "dimensiones" que representan lo que ellos consideran como áreas clave asociadas con el acceso y uso de los servicios financieros: compromiso de los países, la capacidad móvil, entorno normativo y la adopción; esto, según Villasenor, West, y Lewis (2016, p. 2).

Adicionalmente, para Morgan (2015, p. 78), las medidas de inclusión financiera contienen muchos aspectos, como, por ejemplo, el número de sucursales bancarias, cuentas bancarias, cajeros automáticos por cada mil personas, empresas con depósitos o línea de crédito, porcentaje de las pymes con el total de depósitos o línea de crédito, adultos con depósito en instituciones financieras formales y adultos prestatarios.

De otra forma, D’Alcantara y Gautier (2013, p. 125) estudian el papel que los operadores postales pueden jugar en la inclusión financiera; el modelo propuesto se basa en la cuantificación de los costes y los beneficios de la asociación. En otra investigación Demirguc-Kunt y Klapper (2013, p. 279) estudian cómo los adultos usan los sistemas financieros formales e informales para gestionar su día a día de las finanzas y planificar para el futuro; los datos muestran que el 50 por ciento de los adultos de todo el mundo están "bancarizados", es decir, tienen una cuenta en una institución financiera formal.

Ahora bien, cuando se quiere mirar los estudios para cada país, Chakravarty y Pal (2013, p. 813) examinan los efectos de las principales políticas de la banca en 
la inclusión financiera en la India durante los años 1972-2009, y utilizan técnicas de econometría de datos de panel. Para este mismo país, Gupte, Venkataramani, y Gupta (2012, p. 133) se centran en un cálculo de indicadores tradicionales a partir de los datos del Banco Mundial.

China también ha sido objeto de estudio, Fungácová y Weill (2015, p. 296) analizaron la inclusión financiera mediante los indicadores del Banco Mundial, y encontraron un mayor uso de las cuentas de ahorro formal y un uso menos frecuente del crédito.

En Kenia, Johnson y Arnold (2012, p. 719) estudiaron la situación de factores socio-económicos, demográficos y geográficos asociados con el uso de servicios financieros formales, semiformales e informales entre 2006 y 2009, mediante la técnica de regresión de Probit.

De otra manera, Diniz, Birochi, y Pozzebon (2012, p. 484) estudiaron el caso de la inclusión financiera en Autazes, condado de Brasil en la región amazónica no atendido por la banca hasta el año 2002; los datos fueron categorizados mediante el software NVIVO para su condensación.

Para el caso colombiano, mediante el Decreto 3078 de 2006 se crea el Programa de Inversión Banca de las Oportunidades, y se ponen en práctica las recomendaciones expuestas en el documento Conpes 3424 de 2006.

La Banca de las Oportunidades -BdO- y la Superintendencia Financiera de Colombia -SFC-son quienes tienen la responsabilidad de generar y publicar cada año los informes de inclusión financiera. En la primera versión de este informe acopian información desde el año 2008 y hasta el año 2011, y BdO y SFC (2012, p. 14) definen la inclusión financiera como "el acceso y utilización de servicios financieros formales por parte de las actividades económicas y de la población", acogen la propuesta de la AFI respecto a las dimensiones de la inclusión financiera y presentan como método de medición dos indicadores manejados de manera global:

- De acceso: puntos de contacto y cobertura geográfica de los puntos de acceso.

- De uso: indicador de bancarización, productos financieros pasivos, productos financieros activos y canales transaccionales.

Para el año 2012, Bdo y SFC (2013, p. 17) manejan los mismos indicadores e incluyen un aparte sobre protección al consumidor financiero.

En el tercer informe, continúan con los mismos indicadores y adicionan dos tipos de análisis: por puntos de contacto y por tipo de institución financiera; estas 
últimas son analizadas de manera global y estudian 236: bancos, compañías de financiamiento, cooperativas financieras, cooperativas de ahorro y crédito y organizaciones no gubernamentales -ONG- micro-crediticias (BdO y SFC, 2014, p. 31).

En este contexto, se esquematiza el hecho de que en el sistema financiero colombiano hay dos organismos oficiales que supervisan los agentes que desarrollan actividades financieras:

1) La Superintendencia Financiera de Colombia, con las siguientes funciones principales (SFC, 2016): ejerce el control, la inspección y la vigilancia de las personas que realizan actividades aseguradoras, bursátiles, financieras y cualquier otra actividad relacionada con la inversión, el manejo y el aprovechamiento de recursos captados del público en general con el fin de preservar su confianza, estabilidad y seguridad, así como organizar, promover y desarrollar el mercado de valores colombiano y proteger a los asegurados, a los inversionistas y a los ahorradores. Las entidades vigiladas por esta entidad son las compañías de seguros, los bancos, las cooperativas financieras, las compañías de financiamiento y las cooperativas financieras.

2) La Superintendencia de Economía Solidaria (SES, 2016), la cual ejerce la inspección y el control de las cooperativas de ahorro y crédito, para proteger los intereses de todos los asociados de las diferentes organizaciones de economía solidaria, los intereses de los terceros y de la comunidad en general.

La diferencia fundamental entre estas dos entidades es el tipo de instituciones financieras que vigilan; la SFC vigila a

- Bancos: institución financiera que tiene como principal función captar recursos del público mediante depósitos a término y a la vista, para de nuevo colocarlos en forma de descuentos, préstamos, anticipos u otras transacciones activas de crédito.

- Compañías de financiamiento: instituciones cuya principal función consiste en captar dineros a término, con el objeto fundamental de adelantar operaciones de crédito para dar vía libre a la comercialización de servicios y bienes, mediante operaciones del denominado leasing o arrendamiento financiero.

- Cooperativas financieras: organismos cooperativos especializados cuya función principal consiste en adelantar actividades financieras, para ofrecer sus servicios a terceros no asociados.

Y la SES vigila a las cooperativas de ahorro y crédito que son organismos cooperativos especializados con la principal función de realizar actividades financieras de manera exclusiva con sus asociados. 
Para el año 2014 fueron objeto de estudio 229 entidades de los mismos tipos que para el año anterior, según BdO y SFC (2015, p. 16); además, se desplegó un resumen de los resultados en lo referente a inclusión financiera, que analiza la encuesta de demanda Global Findex 2014 y el reporte denominado Microscopio 2014.

De otra manera, en la investigación realizada por Center for Financial Inclusion (2015, p. 21) mediante una metodología de mesa redonda con expertos, se analiza la inclusión financiera en Colombia y el envejecimiento de la población en noviembre de 2014. Esta investigación estableció oportunidades para vincular los créditos de empresa, los créditos de consumo, las transferencias, los seguros y los productos de ahorro a largo plazo con las pensiones, con el objetivo de ampliar el abanico de herramientas financieras ofrecidas a las personas mayores, pero evidenció falta de infraestructura en los servicios financieros, en especial en áreas rurales.

Como conclusión de todo lo expuesto con anterioridad se tiene, por una parte, que la inclusión financiera es un tema de gran importancia tanto en el ámbito nacional como en el internacional; y por otra parte, que diferentes instituciones e investigadores se han ocupado en generar mediciones mediante diferentes tipos de indicadores, pero siempre indicadores tradicionales que se manejan de manera global y no por dependencia o entidad. De tal manera que esta investigación presenta otro ángulo de análisis, pues estudia la situación de la inclusión financiera en Colombia desde un diferente punto de vista: desde la eficiencia relativa; las entidades financieras son el objeto específico, no global, de análisis.

\section{METODOLOGÍA}

\subsection{Data Envelopment Analysis (DEA)}

Es una metodología no paramétrica de programación lineal, que evalúa la eficiencia relativa de unidades organizacionales que toman decisiones (DMU por su término en inglés Decision Making Unit) a partir de la generación de una frontera eficiente mediante la ubicación de los índices relativos individuales, cuando no se conoce la función de producción, según Yang et al. (2014, p. 481) la medida relativa se deriva de la medida universal de eficiencia, Salida/Entrada, como plantean Cooper, Seiford y Tone (2000, p. 3).

El DEA o Análisis Envolvente de Datos es una técnica no paramétrica apropiada para comparar un conjunto de DMU para la determinación de la eficacia relativa y, por lo tanto, discriminar entre las eficientes y no eficientes, según Charnes, Cooper y Rhodes (1978, p. 430). En específico, la eficiencia de cada DMU se define como la 
relación entre las salidas ponderadas y las entradas ponderadas y, por lo tanto, se obtiene mediante la resolución de un problema de programación lineal con el fin de determinar el correcto conjunto de pesos que maximiza (Dotoli et al., 2015, p. 104).

Una de las fortalezas de DEA es que establece el valor numérico de la ineficiencia, según Cook, Kress y Seiford (1993, p. 135). Esta metodología permite, por una parte, evaluar el desempeño de las unidades, y por la otra, identificar su posición competitiva en comparación con otras DMU, según Costantino et al. (2013, p. 851).

DEA fue en un inicio introducido con el modelo CRS (Constant Return to Scale, también denominado CCR por sus autores); después de algunos años de investigación aparece el modelo VRS (Variable Return to Scale, también denominado BCC), el cual admite estudiar retornos no solo constantes (Ghasemi et al., 2015, p. 148).

\subsection{Modelo CRS}

Considere un conjunto compuesto por $n$ DMU denotadas como $\operatorname{DMU}_{j}(j=1, \ldots, n)$ las cuales utilizan recursos $x_{i j}(i=1, \ldots, m)$ y generan $s$ salidas $y_{r j}(r=1, \ldots, s)$, y parta del hecho de que los multiplicadores $\mathrm{v}_{\mathrm{i}}, \mathrm{u}_{\mathrm{r}}$ asociados con $i$ entradas y $r$ salidas, respectivamente, son conocidos. Entonces, en específico, si la $\mathrm{DMU}_{0}$ está bajo estudio, según Rodríguez (2011, p. 74) este modelo está dando la solución del problema de programación fraccional para la medida de eficiencia de esa $\mathrm{DMU}_{0}$ así:

$$
e_{0}=\max \sum_{r} \mu_{r} y_{r o} / \sum_{i} v_{i} x_{i 0}
$$

Sujeto a:

$$
\begin{aligned}
& \sum_{r} \mu_{r} y_{i}-\sum_{i} v_{i} x_{i j} \leq 0, \text { para todo } j \\
& \mu_{r}, v_{i} \geq \varepsilon, \text { para todo } r, i
\end{aligned}
$$

Donde $\varepsilon$ es un valor no-arquimidian designado estrictamente positivo.

Se aplica la teoría de programación fraccionaria expresada en Charnes y Cooper (1962, p. 67) y se realizan los siguientes cambios de variables:

$$
\begin{aligned}
\mu_{r}=t u_{r} \quad \text { y } \quad v_{i}=t v_{i} & \text { donde: } \\
& t=\left(\sum_{i} v_{i} X_{i 0}\right)^{-l}
\end{aligned}
$$

El problema inicial puede ser transformado en el siguiente modelo de programación lineal:

$$
e_{0}=\max \sum_{r} \mu_{r} y_{r o}
$$


Sujeto a:

\subsection{Modelo VRS}

$$
\begin{aligned}
& \sum_{i} v_{i} x_{i 0}=1 \\
& \sum_{r} \mu_{r} y_{r j}-\sum_{i} v_{i} x_{i j} \leq 0, \text { para todo } j \\
& \mu_{r}, v_{i} \geq \varepsilon, \text { para todo } r, i
\end{aligned}
$$

Según todos los parámetros anteriores, para este modelo se tiene como planteamiento matemático (Rodríguez, 2011, p. 76):

$$
e_{0}^{*}=\max \left[\sum_{r} \mu_{r} y_{r o}-\mu_{o}\right] / \sum_{i} v_{i} x_{i o}
$$

Sujeto a:

$$
\begin{aligned}
& \sum_{r} \mu_{r} y_{r j}-\mu_{o}-\sum_{i} v_{i} x_{i j} \leq 0 \leq 0 \quad j=1, \ldots, n \\
& \mu_{r} \geq \varepsilon, v_{i} \geq \varepsilon, \text { para todo } i, r \\
& \mu_{o} \text { no restringida en signo }
\end{aligned}
$$

Con su equivalente en programación lineal:

$$
e_{0}^{*}=\max \sum_{r} \mu_{r} y_{r o}-\mu_{0}
$$

Sujeto a

$$
\begin{aligned}
& \sum_{i} v_{i} x_{i 0}=1 \\
& \sum_{r} \mu_{r} y_{r j}-\mu_{o}-\sum_{i} v_{i} x_{i j} \leq 0, j=1, \ldots, n \\
& \mu_{r} \geq \varepsilon, v_{i} \geq \varepsilon, \text { para todo } i, r \\
& \mu_{o}, \text { irrestricta }
\end{aligned}
$$

DEA necesita de un doble proceso de optimización para establecer las unidades que conforman la frontera eficiente, de acuerdo con Hatami-Marbini, Emrouznejad y Tavana (2011, p. 458). La medición convencional de DEA se basa en la hipótesis de que los recursos (entradas) deben ser minimizados y los productos (salidas) deben ser maximizados como plantean Mahdiloo et al., (2014, p. 480).

\section{REVISIÓN DE LA LITERATURA ACTUAL}

El sector financiero ha sido estudiado mediante DEA por diferentes autores; sin embargo, no hay investigaciones referentes a inclusión financiera y al estudio de la eficiencia relativa de entidades financieras en este contexto. No obstante, se presentan algunas investigaciones actuales con DEA, en el sector financiero. 
Tanto el sistema financiero como los bancos son los temas más recurrentes en el estudio de la eficiencia relativa mediante método no paramétrico; la investigación realizada por Svitalkova (2014, p. 646) compara la eficiencia de los bancos comerciales de República Checa, Eslovaquia, Austria, Polonia, Hungría y Eslovenia; ellos utilizan los modelos VRS y CRS. Las variables de entrada estudiadas fueron: costos de personal, depósitos y activos fijos; y las de salida: ingresos netos por intereses, créditos totales y provisión de créditos incobrables.

Hay ocasiones en las cuales los datos no están disponibles de manera exacta, por lo que se acude a los modelos DEA difusos; esto fue lo que hicieron Puri y Yadav (2014, p. 6421), quienes consideraron 12 bancos de la India; dos variables de entrada: número de empleados y depósitos totales, y dos variables de salida: créditos no morosos y créditos morosos, siendo esta última una salida no deseada.

Para este país también se realizó un estudio mediante el modelo DEA basado en las holguras, para estimar qué tan bien se utiliza el conjunto de entradas o de salidas. Puri y Prasad (2013, p. 1439) aplicaron el modelo al Banco del Estado de Patiala, con los distritos como DMU.

En China se adelantó esta investigación de los bancos que apoyan la construcción; Zhou et al. (2013, p. 104) utilizaron el modelo de juego de negociación de Nash para la descomposición de la eficiencia en el modelo centralizado de dos etapas DEA, donde todas las salidas de la primera etapa son las únicas entradas de la segunda. Se aplicó este modelo para evaluar la eficiencia de 10 sucursales de los bancos ya mencionados.

También para los bancos islámicos se realizó una investigación mediante DEA por parte de Johnes, Izzeldin y Pappas (2014, p. 96); para comparar los resultados con bancos tradicionales, se utilizó el meta-análisis de frontera (AMF), puesto que este último permite la descomposición de la eficiencia en 2 componentes: eficiencia neta (la eficiencia de los bancos se mide en relación con su propio tipo de frontera) y la eficiencia global (la eficiencia que se refiere a modus operandi).

Ghahraman y Prior (2016, p. 83) realizaron un estudio en Canadá para medir la eficiencia de 79 sucursales bancarias mediante el modelo DEA VRS con orientación a las entradas que ellos denominaron de red, pues agruparon con anterioridad las DMU bajo estudio.

Para Brasil, Wanke y Barros (2014, p. 2340) estudiaron la eficiencia en 40 bancos, mediante un modelo de dos etapas para poder modificar las variables de entrada. Para optimizar ambas etapas con simultaneidad, los autores utilizan el modelo centralizado de eficiencia. 
En Colombia se encuentra la investigación realizada por Sarmiento et al. (2014, p. 12), quienes estudian la eficiencia relativa de 23 bancos comerciales durante 10 años, con los modelos CRS y VRS orientados a entradas y orientados a salidas. Plantean, como entradas, depósitos y costos totales; y como salidas, inversiones y cartera en créditos.

Ahora bien, Piraquete, Piñeros y Mondragón (2013, p. 9) analizan la eficiencia de la banca colombiana entre los años 2000 a 2012; encuentran que las entidades nacionales son más eficientes que las extranjeras. Se estudiaron 18 establecimientos bancarios que fueron comparados mediante el modelo VRS orientado a salidas.

\section{DISEÑO METODOLÓGICO}

Para que un modelo DEA esté definido de manera total, se necesita establecer las DMU, el modelo en específico que se utiliza y las entradas y salidas que hacen parte del modelo.

Delimitación de las DMU: ya que se analiza la eficiencia relativa de la inclusión financiera en Colombia y se trabajan las entidades financieras como objeto específico de estudio; las DMU son los bancos, las compañías de financiamiento y las cooperativas financieras. Las fuentes de información son: la Superintendencia Financiera de Colombia y la Banca de las Oportunidades, de tal manera que para el año 2014 se analizaron 52 DMU: 23 bancos, 23 compañías de financiamiento y 6 cooperativas financieras. Los datos corresponden en un 100 \% a la información extractada de las fuentes ya mencionadas ${ }^{1}$.

Determinación del modelo DEA específico: según lo recomendado por Banker, Charnes y Cooper (1984), dado que no se evidenció la existencia de retornos a escala constantes, se optó por utilizar el modelo DEA VRS.

Delimitación de entradas y salidas: según la información disponible para cada una de las DMU establecidas, se crean cuatro entradas y tres salidas:

a. Entradas: en este caso específico, hace referencia a los recursos que las DMU colocan al servicio de la comunidad y con los cuales facilitan el acceso a los servicios financieros:

- Número de oficinas de atención al público

- Número de cajeros

- Número de datáfonos

- Número de corresponsales bancarios

${ }^{1}$ La investigadora expresa su voluntad de suministrar la base de datos usada en el trabajo. Si es el caso, contactarla a girodriguezl@una.edu.co 
b. Salidas: cuando la comunidad utiliza los recursos mencionados con anterioridad, se obtienen determinados productos; que para este caso específico las DMU los traducen en

- Número de operaciones no monetarias

- Número de operaciones monetarias

- Monto de las operaciones monetarias

Ya teniendo totalmente definido el modelo DEA a resolver, se inicia la solución al doble proceso de optimización planteado anteriormente en la ecuación [4]; el primer proceso resuelve, para cada DMU, los niveles óptimos de cada una de las salidas y entradas, y el segundo proceso relativiza los valores de las soluciones óptimas logradas en el primer proceso, generando la frontera eficiente mediante la resolución del problema de programación lineal, y posicionando cada DMU como eficiente o no. En otras palabras, al resolver el doble proceso de optimización, se obtiene un único indicador, en términos porcentuales, para cada DMU. Esta característica de DEA, el generar un solo indicador analizando multientradas y multisalidas, es una de las fortalezas más importantes de DEA.

\section{RESULTADOS}

Los resultados muestran que de los 23 bancos estudiados, solo 11 son eficientes; de las 23 compañías de financiamiento solo 2 son eficientes, y de las 6 cooperativas financieras ninguna es eficiente; esto significa que solo el $25 \%$ del total de las unidades estudiadas son eficientes, como se muestra en las figuras ly 2.

\section{Figura 1. Resultados eficiencia}

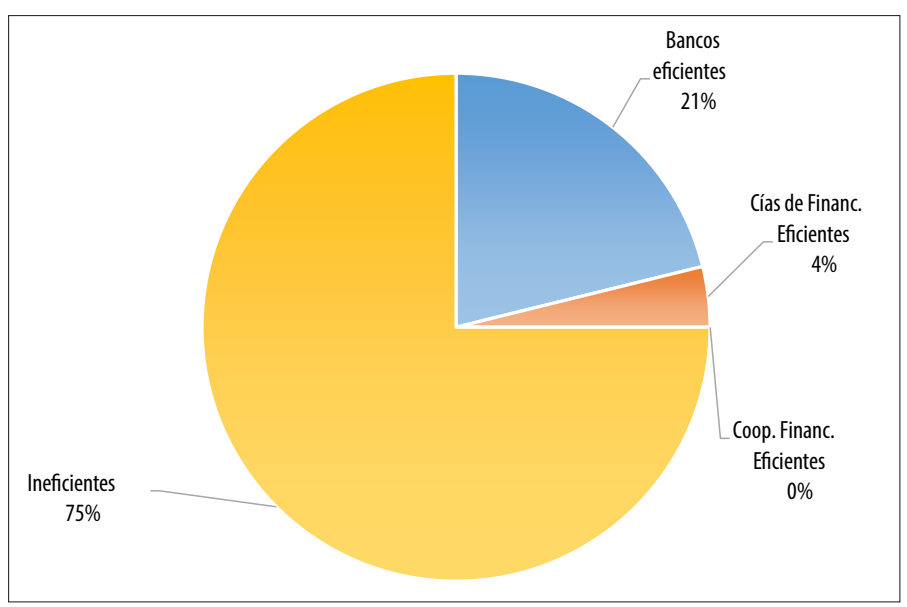

Fuente: elaboración propia 
Figura 2. Unidades eficientes (\%)

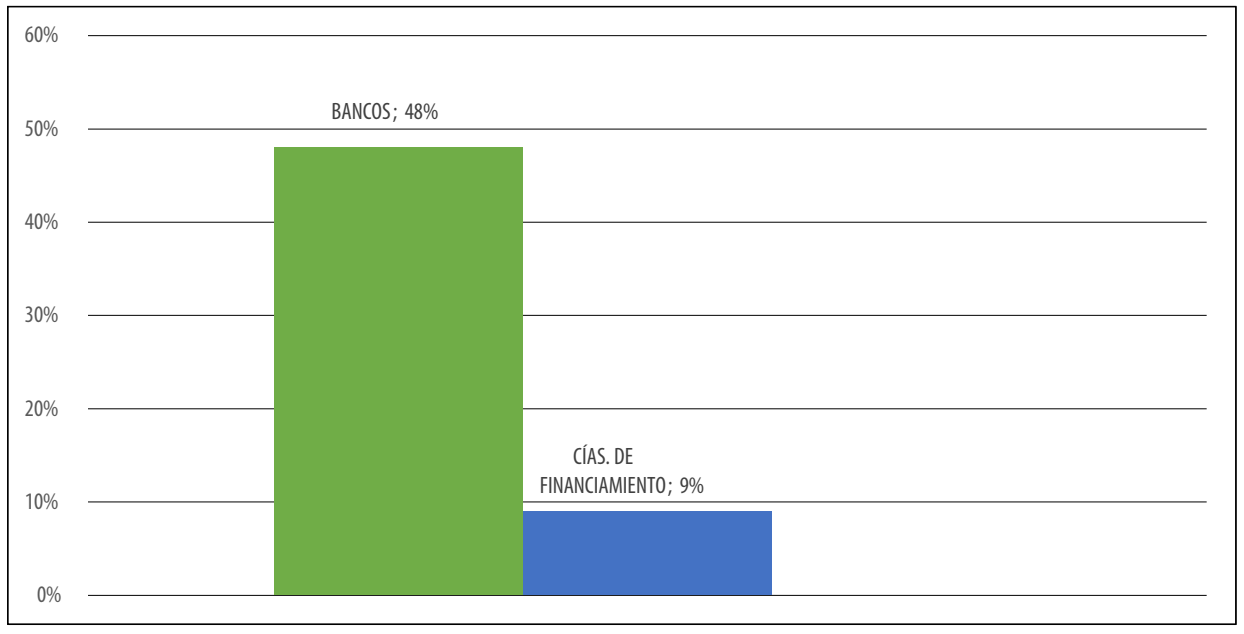

Fuente: elaboración propia

Cuando se analiza el número de unidades por rango de eficiencia, se establece que el rango donde hay mayor cantidad de unidades es el rango del $0 \%$ y el segundo rango es el de $100 \%$, como se muestra en la tabla 1 y en la figura 3.

Tabla 1. Número de unidades por rango de eficiencia

\begin{tabular}{|c|c|c|c|}
\hline \multicolumn{4}{|c|}{ VRS } \\
\hline EF (\%) & \# Unidades & EF (\%) & \# Unidades \\
\hline 100 & 13 & $59,9-40$ & 5 \\
\hline $99,9-80$ & 3 & $39,9-0,1$ & 10 \\
\hline $79,9-60$ & 1 & 0 & 20 \\
\hline
\end{tabular}

Fuente: elaboración propia

Dentro de los bancos analizados, se puede establecer que el banco que es una sociedad de economía mixta del orden nacional, del tipo de las anónimas, sujeta al régimen de empresa industrial y comercial del Estado colombiano es eficiente. Que dentro del grupo de los ineficientes está:

- El banco que mediante su actividad busca contribuir en la superación de las causas estructurales de la pobreza en Colombia.

- El banco que se autodefine como aquel con sentido social que busca apoyar el desarrollo productivo de los emprendedores en la base de la pirámide económica. 
- El banco cuyo accionista principal es una cooperativa médica.

Figura 3. Número de unidades por rango de eficiencia

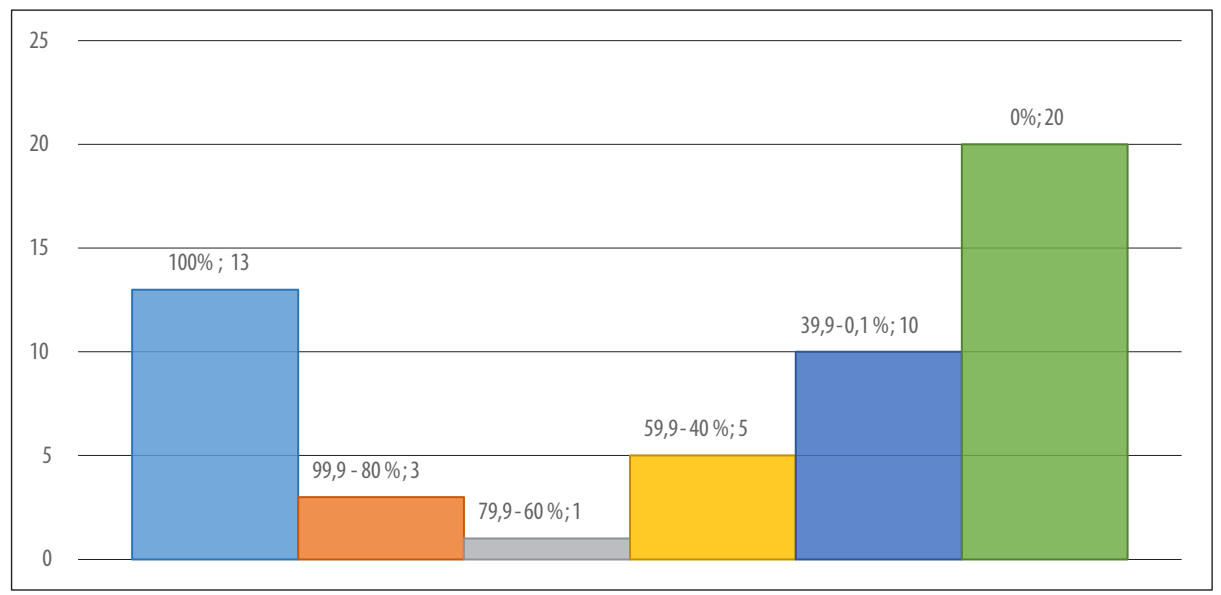

Fuente: elaboración propia

Dado que los bancos representan el $85 \%$ de las unidades eficientes, se hace necesario analizar el comportamiento, en términos de eficiencia relativa, del grupo de las unidades sin los bancos; es decir, de las 23 compañías de financiamiento y de las 6 cooperativas financieras.

Estos resultados muestran que el 38\% de las unidades son eficientes: $10 \mathrm{com}$ pañías de financiamiento y 1 cooperativa financiera (figura 4).

\section{Figura 4. Resultados eficiencia sin bancos}

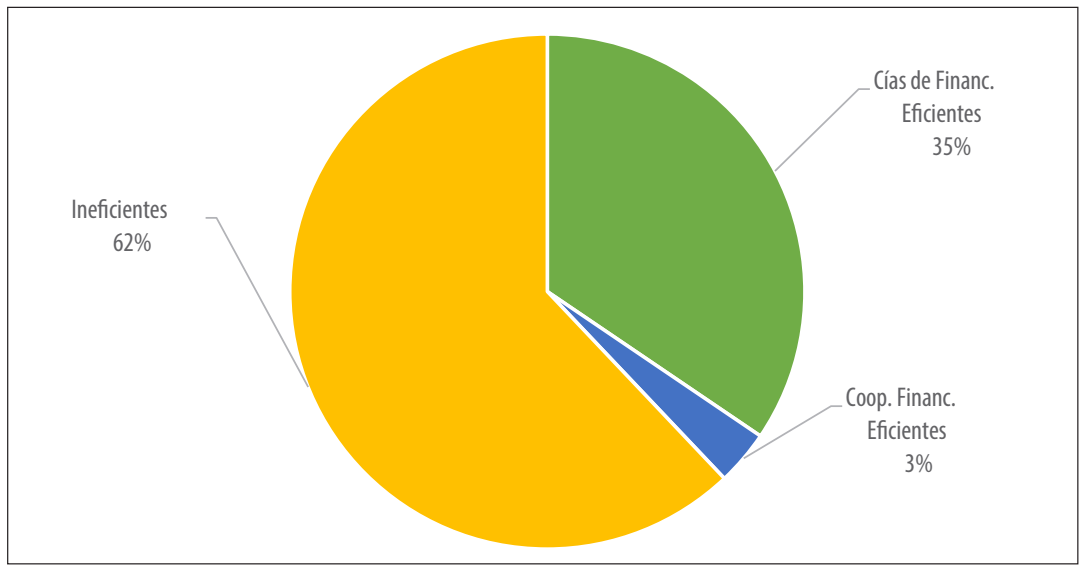

Fuente: elaboración propia 
El análisis por rango de eficiencia se muestra en la figura 5.

Figura 5. Número de unidades por rango de eficiencia sin bancos

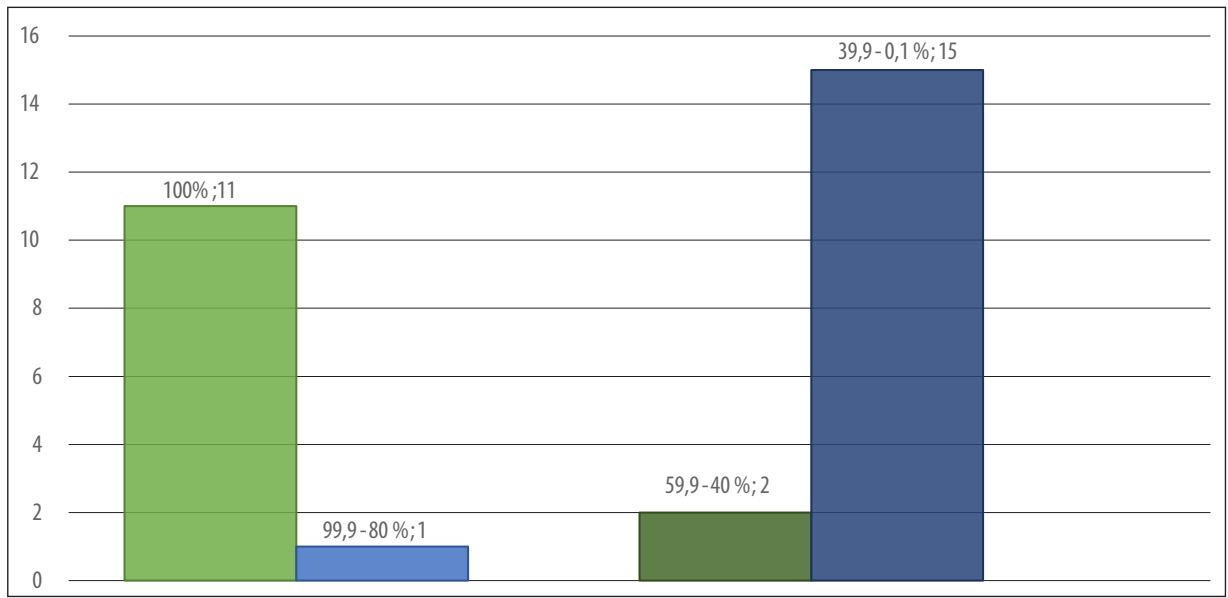

Fuente: elaboración propia

\section{CONCLUSIONES}

En Colombia, los actores que tienen contacto directo con la población en general y que son los llamados a desplegar un papel de primera importancia respecto a operar, apoyar y desarrollar las actividades que tienen referencia con la inclusión financiera son los bancos, las compañías de financiamiento y las cooperativas financieras. Por esta razón, en esta investigación son las unidades a estudiar para establecer la eficiencia relativa de la mencionada inclusión financiera.

De manera global se puede establecer que los resultados, en términos de eficiencia relativa de las instituciones financieras analizadas, no son los mejores; solo el $25 \%$ de ellas son eficientes, lo cual quiere decir que es necesario realizar aún mayores esfuerzos para que las actividades destinadas a mejorar la inclusión financiera efectivamente se concreten.

El 67\% de las instituciones financieras tiene índices de eficiencia relativa por debajo del 60\%. De las compañías de financiamiento solo el 9\% es eficiente.

Todas las cooperativas financieras son ineficientes, pues comparativamente sus niveles de operaciones monetarias, de operaciones no monetarias y el monto de estas últimas no son lo suficientemente altos. De tal manera que es necesario ofrecer servicios en condiciones que los clientes consideren que son comparativamente mejores para que las prefieran y acercarse de mejor manera a los clientes potenciales 
ofreciendo más variados y mejores productos, explicitar el apoyo financiero con que cuentan para demostrar su robustez financiera, e incentivar la apertura de oficinas de atención no solo en las ciudades más importantes; de esta manera contribuirán sólidamente a la inclusión financiera y, adicionalmente, se presume, podrán ser eficientes en el futuro o por lo menos mejorar ostensiblemente sus índices.

Los bancos son las unidades que más contribuyen a los altos índices; hay 13 (57\%) con índices superiores al 80\%. El banco que es una sociedad de economía mixta del orden nacional, del tipo de las anónimas, sujeta al régimen de empresa industrial y comercial del Estado colombiano, es eficiente. El banco que mediante su actividad busca contribuir en la superación de las causas estructurales de la pobreza en Colombia es ineficiente.

En Colombia, los bancos son las instituciones financieras que tradicionalmente ofrecen servicios financieros a los diferentes clientes y, por lo tanto, son los primeros a los cuales la población acude, pues adicionalmente se considera que tienen el suficiente respaldo para adelantar sus operaciones y así lo explicitan en sus innumerables pautas comerciales. Estas son algunas de las razones por las cuales el grupo conformado por los bancos presenta el mayor porcentaje de MDU eficientes. En contraste, tanto las compañías de financiamiento y las cooperativas no están tradicionalmente en el imaginario de las personas del común; adicionalmente, sus pautas publicitarias son bastante más reducidas, de tal manera que no hacen parte de la tradición y no son lo suficientemente agresivas — comercialmente hablandopara que masivamente se conozcan los servicios que ofrecen.

Ahora bien, mirando internamente el comportamiento del grupo de los bancos, esta investigación también establece que el hecho de contar con un mayor número de puntos de contacto con el público no garantiza la eficiencia de este grupo de instituciones financieras; algunos bancos que ostentan grandes números de oficinas y/o cajeros y/o corresponsales bancarios y/o datáfonos tienen resultados bastante bajos, lo que evidencia que no están aprovechando de la mejor manera posible todos estos puntos.

Dado el papel protagónico que desempeñan los bancos en los índices de eficiencia relativa, es necesario conformar un nuevo grupo de unidades a estudiar, compuesto solo por las compañías de financiamiento y las cooperativas financieras. En estos nuevos resultados, se denota que las primeras mejoran ostensiblemente sus resultados, y una cooperativa es eficiente.

No obstante lo anterior, para este nuevo grupo de análisis el 59\% de instituciones financieras tiene índices de eficiencia relativa por debajo del $60 \%$. El $41 \%$ de las unidades tiene índices por encima del $80 \%$ y el 38\% es eficiente. 


\section{BIBLIOGRAFÍA}

Allen, F.; Demirguc-Kunt, A.; Klapper, L. y Martinez, M. (2016). The foundations of financial inclusion: Understanding ownership and use of formal accounts. En: Journal of Financial Intermediation, Vol. 27, p. 1 30.

Angelucci, M.; Karlan, D. y Zinman, J. (2013). Win Some Lose Some? Evidence from a Randomized Microcredit Program Placement Experiment by Compartamos Banco. Cambridge: National Bureau of Economic Research, 68p.

Appleyard, L. (2011). Community Development Finance Institutions (CDFIs): Geographies of financial inclusion in the US and UK. En: Geoforum, Vol. 42, No. 2, p. 250-258.

Banker, R.; Charnes, A. y Cooper, W. (1984). Some Models for Estimating Technical and Scale Inefficiencies in Data Envelopment Analysis. En: Management Sciencie. Vol 30, No. 9, p. $1078-1092$.

Bayero, M. (2015). Effects of Cashless Economy Policy on Financial Inclusion in Nigeria: An exploratory study. En: Procedia - Social and Behavioral Sciences, Vol. 172, p. 49-56.

Bruhn, M. e Inessa, L. (2016). The Economic Impact of Expanding Access to Finance in Mexico. 2p.

Burgess, R. y Pande, R. (2005). Can Rural Banks Reduce Poverty? Evidence from the Indian Social Banking Experiment. En: American Economic Review, Vol. 95, No. 3, p. 780 - 795.

Chakravarty, S. y Pal, R. (2013). Financial inclusion in India: An axiomatic approach. En: Journal of Policy Modeling, Vol 35, No. 5, p. 813-837.

Charnes, A. y Cooper, W. (1962). Programming with linear fractional functional. En: Naval Research Logistics Quarterly, Vol. 9, No. 3-4, p. 67-88.

Charnes, A.; Cooper, W. y Rhodes, E. (1978). Measuring the efficiency of decision making units. En: European Journal of Operational Research, Vol. 2, No. 6, p. 429-444.

Cook, W.; Kress, M. y Seiford, L. (1993). On the Use of Ordinal Data in Data Envelopment Analysis. En: Journal of Operational Research Society, Vol. 44, No. 2, p. 133-140.

Cooper, W.; Seiford, L. y Tone, K. (2000). Data Envelopment Analysis: A Comprehensive Text with Models, Applications, References and DEA-Solver Software. Boston, Massachusetts: Kluwer Academic Publishers, 219p.

Costantino, N.; Dotoli, M.; Falagario, M.; Fanti, M. P.; Mangini, A. M. y Sciancalepore, F. (2013). A hierarchical optimization technique for the strategic design of distribution networks. En: Computers \& Industrial Engineering, Vol. 6, No. 4, p. 849-864.

Cull, L., Demirgüç-Kunt, A. y Lyman, T. (2012). Inclusión y estabilidad financiera: ¿qué demuestran las investigaciones? Washington, DC: CGAP, 4p.

Cull, R.; Ehrbeck, T. y Holle, N. (2014a). La inclusión financiera y el desarrollo: Pruebas recientes de su impacto. Washington, DC, CGAP, 12p.

Cull, R., Ehrbeck, T. y Holle, N. (2014b). Financial Inclusion and Development: Recent Impact Evidence. Washington, DC, CGAP, 12p. 
D'alcantara, G. y Gautier, A. (2013). The postal sector as a vector of financial inclusion. En: Annals of Public and Cooperative Economics, Vol. 84, No. 2, p. 119-137.

Dasgupta, R. (2009). Two Approaches to Financial Inclusion. En: Economic and Political Weekly, Vol. 44, No. 26/27 p. 41-44.

De Koker, L. (2013). Financial Inclusion and Financial Integrity: Aligned Incentives? En: World Development, 44, p. 267-280.

Demirguc-Kunt, A. y Klapper, L. (2013). Measuring Financial Inclusion: Explaining Variation in Use of Financial Services across andwithin Countries. En: Brookings Papers on Economic Activity, Vol. 44, No. 1, p. 279-321.

Diniz, E.; Birochi, R. y Pozzebon, M. (2012). Triggers and barriers to financial inclusion: The use of ICT-based branchless banking in an Amazon county. En: Electronic Commerce Research and Applications, Vol.11, No. 2, p. 484-494.

Dotoli, M.; Epicoco, N.; Falagario, M. y Sciancalepore, F. (2015). A cross-efficiency fuzzy Data Envelopment Analysis technique for performance evaluation of Decision Making Units under uncertainty. En: Computers E Industrial Engineering, Vol. 79, p. 103-114.

Friedline, T. y Rauktis, M. (2014). Young People Are the Front Lines of Financial Inclusion: A Review of 45 Years of Research. En: The Journal of Consumer Affairs, Vol. 48, No. 3, p. 535-602.

Fungácová, Z. y Weill, L. (2015). Understanding financial inclusion in China. En: China Economic Review, Vol. 34, p. 196-206.

García, N.; Grifoni, A.; Lopez, J. y Mejía, D. (2013). Financial Education in Latin America and the Caribbean: Rationale, Overview and Way Forward. OECD working papers on finance, insurance and private pensions, No. 33, 74p.

Ghahraman, A. y Prior, D. (2016). A learning ladder to ward efficiency: Proposing network-based stepwise benchmark selection. En: Omega, Vol. 63, p. 83-93.

Ghasemi, M.; Ignatius, J.; Lozano, S.; Emrouznejad, A. y Hatami-Marbini, A. (2015). A fuzzy expected value approach under generalized data envelopment analysis. En: KnowledgeBased Systems, Vol. 89, p. 148-159.

Gupte, R.; Venkataramani, B. y Gupta, D. (2012). Computation of financial inclusion index for India. En: Procedia - Social and Behavioral Sciences, Vol. 37, p. 133-149.

Gwalani, H. y Parkhi, S. (2014). Financial inclusion - Building a success model in the Indian context. En: Procedia - Social and Behavioral Sciences, Volo. 133, p. 372-378.

Hatami-Marbini, A.; Emrouznejad, A. y Tavana, M. (2011). A taxonomy and review of the fuzzy data envelopment analysis literature: two decades in the making. En: Euro Journal Opertional Research. Vol. 214, No. 3, p. 457-472.

Johnes, J.; Izzeldin, M. y Pappas, V. (2014). A comparison of performance of Islamic and conventional banks 2004-2009. En: Journal of Economic Behavior \& Organization, Vol. 103, p. 93-107.

Johnson, E. y Sherraden, M. (2007). From Financial Literacy to Financial Capability. En: Journal of Sociology and Social Welfare, Vol. 34, No. 3, p. 119-146. 
Johnson, S. y Arnold, S. (2012). Inclusive Financial Markets: Is Transformation Under Way in Kenya? En: Development Policy Review, Vol. 30, No. 6, p. 719-748.

Kapoor, A. (2014). Financial inclusion and the future of the Indian economy. En: Futures, Vol. 56, p. $35-42$.

Kear, M. (2013). Governing Homo Subprimicus: Beyond Financial Citizenship, Exclusion, and Rights. En: Antipode, Vol. 45, No. 4, p. 926946.

Mahdiloo, M.; Tavana, M.; Saen, R. y Noorizadeh, A. (2014). A game theoretic approach to modeling undesirable outputs and efficiency decomposition in data envelopment analysis. En: Applied Mathematics and Computation, Vol. 244, p. 479-492.

Morales, L.; y Yáñez, A. (2006). La bancarización en Chile. En: Serie Técnica de Estudios de la Superintendencia de Bancos e Instituciones Financieras, No. 7, p. $1-18$.

Morgan, P. (2015). Financial Stability and Financial Inclusion. En A. I. Economics, Financial System Stability, Regulation, and Financial Inclusion. Tokyo: Springer, 122p.

Piraquete, J.; Piñeros, J. y Mondragón, L. (2013). Eficiencia en los Establecimientos Bancarios (EB): una aproximación mediante modelos DEA. En: Borradores de Economía. No. 798, 39 p.

Puri, J.; y Prasad, S. (2013). A concept of fuzzy input mix-efficiency in fuzzy DEA and its application. En: Expert Systems with Applications, Vol. 40, No. 5, p.1437-1450.

Puri, J., y Yadav, S. (2014). A fuzzy DEA model with undesirable fuzzy outputs and its application to the banking sector in India. En: Expert Systems with Applications, Vol. 41, No. 14, p. 6419-6432.

Roa, M. (2013). Financial Education and Behavioral Finance: New Insights into the role of Information in financial decisions. En: Journal of Economic Surveys, Vol. 27, No. 2, p. 297-315.

Roa, M. (2014). La Inclusión y la Estabilidad Financieras. Documentos de Investigación 15. México D.F.: CEMLA, 28p.

Rodríguez, G. (2011). Indicadores DEA (Data Envelopment Analysis) de eficiencia y productividad para las actividades de extensión universitaria. Aplicación en la Universidad Nacional de Colombia, Bogotá, Centro Editorial Facultad de Ciencias Económicas Universidad Nacional de Colombia, 262p.

Sarma, M. y Pais, J. (2011). Financial Inclusion and development. En: Journal of International Development, Vol. 23, No. 5, p. 613-628.

Sarmiento, M.; Cepeda, A.; Mutis, H. y Pérez, J. (2014). Nueva Evidencia sobre la Eficiencia de la Banca Colombiana: Una medición con modelos de frontera no-paramétricos. En: Archivos de Economía. Documento 392, 49p.

Svitalkova, Z. (2014). Comparison and Evaluation of Bank Efficiency in Selected Countries in EU. En: Procedia Economics and Finance, Vol. 12, p. 644-653.

Thorat, U. (2006). Financial inclusion and millennium goals. 2006: Reserve Bank of India, 250p.

Vera, C. y Titelman, D. (2013). Financiamiento para el Desarrollo El Sistema Financiero en América Latina y el Caribe. Santiago de Chile: CEPAL, 396p. 
Villacorta, O. y Reyes, J. (2012). Servicios financieros para las mayorías, La inclusión financiera en México. En: Revista mbs, No. 2, p. 5-21.

Villasenor, J., West, D., y Lewis, R. (2016). The 2016 brookings financial and digital inclusion project report. Washington: Center for Technology Innovation at Brookings, 164p.

Wanke, P. y Barros, C. (2014). Two-stage DEA: An application to major Brazilian banks. En: Expert Systems with Applications, Vol. 41, No. 5, p. 2337-2344.

Yang, M.; Li, Y.; Chen, Y. y Liang, L. (2014). An equilibrium efficiency frontier data envelopment analysis approach for evaluating decision-making units with fixed-sum outputs. En: European Journal of Operational Research, Vol. 239, No. 2, p. 479-489.

Zhan, M. y Sherraden, M. (2011). Assets and Liabilities, Educational Expectations, and Children's College Degree Attainment. En: Children and Youth Services Review, Vol.33, No. 6, p. 846-854.

Zhou, Z.; Sun, L.; Yang, W. y Ma, C. (2013). A bargaining game model for efficiency decomposition in the centralized model. En: Computers \& Industrial Engineering, Vol. 64, No. 1, p. 103-108.

\section{OTRAS REFERENCIAS BIBLIOGRÁFICAS}

AFI -Alliance for Financial Inclusion- (2011). Measuring Financial Inclusion. Core Set of Financial Inclusion Indicators. Bangkok: Financial Inclusion Data Working Group, Alliance for Financial, 20p.

AFI -Alliance for Financial Inclusion- (2015). The Maya Declaration Progress Report Commitments Into Action. Kuala Lumpur: AFI, 52p.

AFI-Alliance for Financial Inclusion- (2016a). A Financial Inclusion Index to measure the progress of Financial Inclusion. . Kuala Lumpur: Alliance for Financial Inclusion, 8p.

AFI -Alliance for Financial Inclusion- (2016b). Alliance for Financial Inclusion, 4p.

BCE-Banco Central del Ecuador- (2012). Inclusión Financiera Aproximaciones teóricas y prácticas. Quito: Banco Central del Ecuador (BCE), 112p.

BCB -Banco Central do Brasil- (2011). Relatório de Inclusão. Brasília: Banco Central do Brasil, 183p.

Banco Mundial (2016). Base de datos Global Findex. Obtenido de Global Financial Development Report 2014, 24p.

BdO y SFC -Banca de las Oportunidades y Superintendencia Financiera de Colombia- (2012) Reporte de Inclusión Financiera 2011, Bogotá, BdO y SFC, 154p.

Bdo y SFC -Banca de las Oportunidades y Superintendencia Financiera de Colombia- (2013) Reporte de Inclusión Financiera 2012, Bogotá, BdO y SFC, 151p.

BdO y SFC -Banca de las Oportunidades y Superintendencia Financiera de Colombia- (2014). Reporte de Inclusión Financiera 2013, Bogotá, BdO y SFC, 204p.

BdO y SFC -Banca de las Oportunidades y Superintendencia Financiera de Colombia- (2015) Reporte Inclusión Financiera 2014, Bogotá, BdO y SFC, 200p.

Center for Financial Inclusion (2015). Inclusión financiera y envejecimiento: una oportunidad. MetLife Foundation, 40p. 
CFI -Committee on Financial Inclusion- (2008). Report of the Committee on Financial inclusion. New Delhi, 167p.

CGAP - Grupo Consultivo de Ayuda a los Pobres- (2011). Global Standard-Setting Bodies and Financial Inclusion for the Poor: Towards Proportionale Standards and Guidance. CGAP, 62p.

CNIF -Consejo Nacional de Inclusión Financiera de México- (2012). Reporte de Inclusión Financiera 4. México D.F.: Consejo Nacional de Inclusión Financiera, 112p.

GPFI -Global Parnership for Financial Inclusion- (2016). G 20 Financial Inclusion Indicators. Hangzhou: GPFI, 8p.

SES -Superintendencia de Economía Solidaria- (2016). Superintendencia de Economía Solidaria.

SFC -Superintendencia Financiera de Colombia- (2016). Superintendencia Financiera de Colombia.

The Economist Intelligence Unit. (2014). Microscopio Global 2014: Análisis del entorno para la inclusión financiera. New York: EIU, 91 p.

World Bank Group (2015). 2015 The little data book on Financial Inclusion. Washington: Worl Bank Group, 176p. 


\section{ANEXO}

Tabla 2. Grado de eficiencia por institución

\begin{tabular}{|l|c|}
\hline \multicolumn{1}{|c|}{ Institución financiera } & EF (\%) \\
\hline Bancolombia & 100 \\
\hline Banco Davivienda & 100 \\
\hline Banco de Bogotá & 100 \\
\hline Banco de Occidente & 100 \\
\hline Banco Agrario de Colombia & 100 \\
\hline Banco Popular & 100 \\
\hline Citibank Colombia & 100 \\
\hline Banco Corpbanca & 100 \\
\hline Banco Falabella & 100 \\
\hline Banco GNB Sudameris & 100 \\
\hline Banco GNB & 100 \\
\hline Compañía de Financiamiento TUYA & 100 \\
\hline Serfinansa Compañía de Financiamiento & 100 \\
\hline Banco Colpatria & 88,85 \\
\hline Banco AV Villas & 83,72 \\
\hline Leasing Bancolombia & 83,65 \\
\hline BBVA Colombia & 71,97 \\
\hline Banco Caja Social & $10,64,29$ \\
\hline Banco Coomeva & 55,63 \\
\hline Ripley Compañía de Financiamiento & 53,29 \\
\hline Banco Pichincha & 47,42 \\
\hline Confiar Cooperativa Financiera & 46,51 \\
\hline Cotrafa Cooperativa Financiera & 35,08 \\
\hline Banco de las Microfinanzas - Bancamía & 27,57 \\
\hline Coltefinanciera & 15,2 \\
\hline Banco WWB & 1,06 \\
\hline Cooperativa Financiera John F. Kennedy & 10,6 \\
\hline Giros E Finanzas & 100 \\
\hline Macrofinanciera & 100 \\
\hline Financiera Juriscoop Cía. Financiamiento & 100 \\
\hline
\end{tabular}




\begin{tabular}{|l|c|}
\hline \multicolumn{1}{|c|}{ Institución financiera } & EF (\%) \\
\hline Finamerica & 3,2 \\
\hline Cooperativa Financiera de Antioquia & 0,54 \\
\hline Banco Procredit Colombia & 0 \\
\hline Banco Finandina & 0 \\
\hline Banco Cooperativo Coopcentral & 0 \\
\hline Banco Santander de negocios Colombia & 0 \\
\hline Dann Regional Compañía de Financiamiento & 0 \\
\hline OIColombia & 0 \\
\hline G. M. A. C. Financiera de Colombia & 0 \\
\hline Mi Plata & 0 \\
\hline Financiera Pagos Internacionales & 0 \\
\hline la Polar & 0 \\
\hline Internacional Compañía de Financiamiento & 0 \\
\hline Credifamilia & 0 \\
\hline Credifinanciera & 0 \\
\hline La Hipotecaria Compañía de Financiamiento & 0 \\
\hline Leasing Corficolombiana & 0 \\
\hline Factoring Bancolombia & 0 \\
\hline Leasing Bancoldex & 0 \\
\hline Leasing Bolivar & 0 \\
\hline Coofinep Cooperativa Financiera & 0 \\
\hline Financiera Juriscoop Cooperativa Financiera & \\
\hline
\end{tabular}

\section{Fuente: elaboración propia}

